ORIGINAL ARTICLE

\title{
Unnecessary laparotomy by using physical examination and different diagnostic modalities for penetrating abdominal stab wounds
}

\author{
C Ertekin, H Yanar, K Taviloglu, R Güloglu, O Alimoglu
}

Emerg Med J 2005;22:790-794. doi: 10.1136/emj.2004.020834

Background: The modern management of penetrating abdominal trauma has decreased the incidence of unnecessary laparotomy by using selective non-operative management protocols. However, the real benefits of physical examination and different diagnostic methods are still unclear.

See end of article for Methods: From January 2000 to April 2003, we prospectively collected data on 117 patients with authors' affiliations penetrating stab wounds to the thoracoabdominal, anterior abdominal, and back regions who had nonoperative management. Clinical examination was the primary tool to differentiate those patients requiring ....................

Correspondence to: Dr C Ertekin, Istanbul University, Istanbul Faculty of Medicine, Trauma and Emergency Medicine Service, 34390, Çapa, Istanbul; cemalettin@ ertekin.info operation. Findings of physical examination, ultrasound, computed tomography, endoscopy, echocardiography, diagnostic peritoneal lavage, and diagnostic laparoscopy were reviewed. The number of therapeutic, non-therapeutic, and negative laparotomies were recorded.

Results: Non-operative management was successful in 79\% of patients. There were 11 early (within 8 hours of admission) and 14 delayed (more than 8 hours after admission) laparotomies performed, depending on the results of various diagnostic procedures. Non-operative management failed in $21 \%$ of patients, and the rate of non-therapeutic laparotomy in early and delayed laparatomy groups was $9 \%$ and $14 \%$ respectively. There was no negative laparatomy.

Accepted for publication 22 December 2004

Conclusions: The use of physical examination alone and/or together with different diagnostic methods allows reduction of non-therapeutic laparotomies and elimination of negative laparatomies.

A lthough the presence of classic indications such as shock and peritoneal irritation indicates laparotomy after penetrating abdominal stab wounds, the trend has moved in the past two decades from mandatory exploration to selective approach. ${ }^{1-6}$ Mandatory laparotomy for penetrating abdominal stab wounds leads to unnecessary operations in $38-40 \%$ of patients, and postoperative morbidity ranges from $3 \%$ to $16 \% .^{78-11}$

The decision as to when to operate on a patient with a penetrating abdominal stab wound is a continuing challenge. Additional diagnostic procedures have been advocated to enhance the sensitivity and specificity of clinical judgment alone in evaluating patients. Several diagnostic methods, including ultrasound (US), computerised tomography (CT), diagnostic peritoneal lavage (DPL), diagnostic laparoscopy (DL), endoscopy, echocardiography and intravenous pyelogram (IVP), may be used in insignificant injuries on carefully selected patients.

The aim of this study was to evaluate the benefits of physical examination and diagnostic methods in the nonoperative management of penetrating abdominal stab wounds, and the effect of timing of the operation.

\section{MATERIALS AND METHODS}

As an initial approach, the standard resuscitative protocol performed for all patients care as approved by Advanced Trauma Life Support guidelines was used. ${ }^{12}$ Preoperative antibiotics such as cefozoline sodium $(20 \mathrm{mg} / \mathrm{kg}$ ) were administered intravenously, and all patients received tetanus toxoid. Nasogastric tube and Foley catheter were inserted in selected patients.

Wound exploration was performed to wounds inferior to the costal margins and between the mid-axillary lines. For one or two stab wound sites at the anterior abdomen, wound exploration was performed, while laparoscopy was considered for patients with three or more wound sites at the anterior abdomen. Patients presenting with stab wounds at other locations such as the back were evaluated separately. Following skin preparation with an antiseptic solution, a local anesthetic agent was applied to the wound. A retractor was inserted through the entry of the wound and peritoneal or posterior fascial penetration investigated. The fascial defect from the stab wound was closed in all cases to prevent recurrent herniation or evisceration. Symptomatic but haemodynamically stable patients were admitted for $48 \mathrm{~h}$ for clinical observation, and patients were discharged when feeding was normal and if there was clinical improvement.

Herniation of omentum or bowel was not accepted as an indication for immediate operation. These patients were managed selectively after excision of non-viable omentum or reduction of the herniated organs into the abdomen.

\section{Categorisation: wound site}

Patients were categorised into three groups based on the location of their wound sites:

(a) The anterior abdomen was defined as the region between anterior costal margins superiorly and pubic symphysis inferiorly, between midaxillary lines. Anterior flank injuries were also included in this group.

(b) Thoracoabdomen was defined as the region between the midaxillary lines laterally, the fourth intercostal spaces above, and anterior costal margins below.

(c) The back was defined as the region from the inferior scapular tip to the gluteal folds, between the midaxillary

Abbreviations: $\mathrm{CT}$, computerised tomography; $\mathrm{DL}$, diagnostic laparoscopy; DPL, diagnostic peritoneal lavage; IVP, intravenous pyelogram; US, ultrasound 
lines. Posterior flank injuries were also included in this group.

Clinical examination was the primary tool to differentiate the patients requiring surgery. Further special diagnostic procedures were used before early and delayed laparotomies except immediate laparotomies.

\section{Methods of evaluation and special diagnostic procedures \\ Serial physical examinations, white blood cell counts, and rectal/axillary temperature measures All patients underwent serial physical examinations and close observations, white blood cell counts, and rectal/axillary temperature measures every 4 hours after hospitalisation. The onset of peritoneal signs, leucocytosis, or rectal-axillary temperature difference of $>1{ }^{\circ} \mathrm{C}$ warranted further investiga- tion.}

\section{Diagnostic peritoneal lavage and diagnostic laparoscopy}

DPL, using the open technique at the infraumbilical ring, ${ }^{13}$ was used for haemodynamically stable patients whose physical examinations were not reliable because of alcohol or narcotic usage or unconsciousness. In the absence of intestinal fluid, $15 \mathrm{ml} / \mathrm{kg}$ of normal saline up to maximum of 1 litre was instilled. The returning lavage fluid was analysed for amylase and alkaline phosphatase levels and presence of bacteria. Any of the following were considered to be a positive result: amylase activity $\geqslant 20 \mathrm{IU} / \mathrm{l}$, alkaline phosphatase activity $\geqslant 3 \mathrm{IU} / \mathrm{l}$, urea and creatinine levels higher than blood values, or the presence of bacteria and intestinal fluid. The amount of blood was not considered as an indication for laparotomy in haemodynamically stable patients. Afterwards, the abdominal cavity was washed with $0.9 \% \mathrm{NaCl}$ until the lavage fluid appeared to be cleared.

The main indications for DL were: haemodynamically stable patients with left thoracoabdominal stab wounds, uncertain findings of peritonitis in patients with anterior stab wounds, and presence of omental or bowel herniation in selected patients.

\section{Scanning and other investigations}

All patients with anterior abdomen and thoracoabdominal injuries were evaluated by ultrasound except those underwent mandatory laparotomy. Ultrasound (particularly focused abdominal sonography for trauma; FAST) was used to evaluate Morrison and Douglas pouches, perisplenic and perihepatic regions for presence or absence of fluid, and solid organ injuries. Sonograms were obtained by radiology residents in emergency department in the presence of surgical staff.

Triple contrast CT was used for the differential diagnosis of stab injuries of the back region, and is our routine practice. Contrast material was administered by intravenous, oral, and rectal routes.

Colonoscopy was performed in patients with penetrating injuries of the back, and gastroscopy was performed in cases of epigastric injury.

For left thoracoabdominal injuries, echocardiography was performed to evaulate the pericardium for the presence or absence of fluid.

IVP was used in patients with microhaematuria to rule out a urinary system injury. It also enabled the physician to predict the functional status of the kidneys and urinary bladder.

\section{Categorisation: timing}

Patients were also classified based on the timing of surgery: (a) Immediate laparotomies included patients who were in shock on admission, required emergent intubation, had failure of reduction of the herniated bowel or in whom there had been aspiration of blood from the nasogastric tube. These patients were excluded from the study;

(b) Early laparotomies were usually performed because of sudden drop in haematocrit value and/or blood pressure in the first 8 hours of admission, but increase in white blood cell count and temperature also made surgery mandatory. DPL and DL were the most common diagnostic procedures used in this group

(c) Delayed laparotomies were those performed more than 8 hours after admission. Increases in white blood cell count and temperature, appearance of new abdominal tenderness, and generalised abdominal pain were among the indications for delayed laparotomies.

\section{Laparotomy classification}

A laparotomy was defined as "therapeutic" if intra-abdominal injuries requiring repair were found, and was defined as "nontherapeutic" if the injuries did not require intervention (for example, non-bleeding liver and/or spleen lacerations, serosal bowel injuries, or non-expanding retroperitoneal haematomas). When no intra-abdominal injuries were found, the laparotomy was considered as "negative". All non-therapeutic and negative laparotomies were defined as "unnecessary laparotomies". Selective management was deemed to have failed when the patients underwent early or delayed laparotomies.

\section{Data collection and statistical analysis}

All clinical data were collected prospectively and entered into a study database. Severity of injury was assessed by the Penetrating Abdominal Trauma Index. ${ }^{14}$ Data evaluated included patient demographics, location of injury, laboratory values, physical findings, results of diagnostic methods, duration of observation, treatment plans, unnecessary laparotomies, and complications. For statistical analyses, SPSS for Windows (release 10.0.1; SPSS Inc., Chicago, IL, USA) was used.

\section{RESULTS}

From January 2000 to April 2003, 294 consecutive patients with abdominal stab wounds were admitted to our Level I trauma centre. In total, 165 patients with no penetration of peritoneum at local wound exploration and 12 patients who were operated immediately due to shock, irreduction of organs, or aspiration of blood via the nasogastric tube on admission were excluded from the trial. Details of the 12 patients who were operated on immediately were given in table 1 . The remaining $117(40 \%)$ patients with stab wounds penetrating the peritoneum were candidates for non-operative management protocol and were evaluated prospectively. There were 113 men (96.5\%) and 4 women (3.5\%); average age was 28.3 years (range 15 to 52 ). Locations of the stab wounds were: anterior abdomen in $76(65 \%)$ patients, thoracoabdomen in $24(20.5 \%)$, and back region in 17 (14.5\%). There were 92 patients (79\%) discharged from the hospital without surgery, while for the remaining 25 patients $(21 \%)$, non-operative management failed and they were taken to the operating theatre. In the first 8 hours of admission, 11 patients $(9.4 \%)$ were taken to the operating theatre for "early" laparotomies, and "delayed" laparotomies were performed on 14 patients (11.9\%) (table 2 ).

The rate of unnecessary laparotomies among all the operated patients including the immediate operations was $16 \%$ while the rates in early and delayed laparotomy groups were $9 \%$ and $14 \%$ respectively $(p=1.0$, Fisher's exact test). 
Table 1 Immediate laparotomies.

\begin{tabular}{lllll}
\hline $\begin{array}{l}\text { Patient } \\
\text { no. }\end{array}$ & $\begin{array}{l}\text { Diagnostic criteria } \\
\text { for operation }\end{array}$ & Injured organs & PATI & $\begin{array}{l}\text { Morbidity } \\
\text { and mortality }\end{array}$ \\
\hline 1 & Hypovolaemic shock & Liver, spleen, transverse colon & 24 & Pneumonia \\
2 & Hypovolaemic shock & Right iliac vein & 16 & DVT \\
3 & Hypovolaemic shock & Spleen, abdominal wall & 9 & - \\
4 & Hypovolaemic shock & Small bowel, transveres colon & 26 & Evisceration \\
5 & Hypovolaemic shock & Liver & 12 & - \\
6 & Hypovolaemic shock & Liver, small bowel, right colon & 33 & Exitus \\
7 & Hypovolaemic shock & SMV, IEA, IEV, small bowel & 24 & Exitus \\
8 & Hypovolaemic shock & Small bowel, left colon, ureter & 16 & - \\
9 & Irreduction of organ & Small bowel serosa & 2 & - \\
10 & Irreduction of organ & Caecum, small bowel & 14 & - \\
11 & Irreduction of organ & Small bowel & 6 & - \\
12 & Aspiration of blood from & Stomach, small bowel & 13 & - \\
& nasogastric tube & & \multicolumn{2}{l}{} \\
\hline
\end{tabular}

PATI, Penetrating Abdominal Trauma Index; SMV, superior mesenteric vein; IEA, inferior epigastric artery; IEV, inferior epigastric vein.

There was no negative laparotomy in the whole series (table 3).

In total, 19 patients (16\%) were admitted to the emergency department with evisceration after stabbing. Sixteen patients had eviscerated omentum, and three had eviscerated small bowel. Of these patients, only one required laparotomy and he was operated 16 hours after admission because of the presence of bile, revealed by DL. The remaining 18 patients were discharged after 48 hours of observation.

Of the total 117 patients included in the study, $14(12 \%)$ underwent DPL. Indications for DPL were: the findings of uncertain peritonitis $(n=2)$, unconsciousness because of head trauma $(\mathrm{n}=1)$, and haemodynamic instability with sudden drop of haematocrit value $(n=1)$ in the early laparotomy group, and progressing abdominal tenderness with increasing leucocyte levels $(n=4)$ and high temperature $(n=2)$ in the delayed laparotomy group. The findings of DPL were: intestinal fluid $(\mathrm{n}=2)$ and aspiration of $\sim 1$ litre of blood (one haemodynamically instable patient) in the early laparotomy group, and intestinal fluid $(n=5)$ and elevated urea and creatinine levels in lavage fluid $(n=1)$ in the delayed laparotomy group. DPL performed because of progressing abdominal tenderness in four patients was considered negative, and these patients were discharged after 24 hours of observation.

DL was performed on 38 patients (32\%), 24 of whom had thoracoabdominal injuries (20 patients on the left side and 4 on the right). The patients with right sided injuries also had multiple anterior abdominal stab wounds. Of the remaining 14 patients, 5 had anterior abdominal injuries, and all of them had signs of uncertain peritonitis (table 2).

In our non-operative management protocol, US was the most commonly used additional diagnostic test. Of the 100 performed US scans, free fluid was demonstrated in 23 patients, spleen injuries in 18, and liver injuries in 12 .

CT examination was used for 12 of the 17 patients sustaining injuries to the back. A patient with retroperitoneal haematoma was observed non-operatively; colonoscopy and

Table 2 Early and delayed laparotomies

\begin{tabular}{|c|c|c|c|c|c|c|c|}
\hline $\begin{array}{l}\text { Patient } \\
\text { ID }\end{array}$ & $\begin{array}{l}\text { Classification } \\
\text { of laparotomy }\end{array}$ & $\begin{array}{l}\text { Diagnostic } \\
\text { procedures } \\
\text { for operation }\end{array}$ & $\begin{array}{l}\text { Wound } \\
\text { site }\end{array}$ & $\begin{array}{l}\text { Delay } \\
\text { (hours) }\end{array}$ & $\begin{array}{l}\text { Description } \\
\text { of injuries }\end{array}$ & PATI & $\begin{array}{l}\text { Morbidity } \\
\text { and mortality }\end{array}$ \\
\hline \multicolumn{8}{|c|}{ Early laparotomies } \\
\hline 1 & $\mathrm{~T}$ & DPL & AA & 4 & Small bowel & 6 & - \\
\hline 2 & $\mathrm{~T}$ & DPL & $\mathrm{AA}$ & 8 & Transverse colon & 15 & - \\
\hline 3 & $\mathrm{~T}$ & DPL & AA & 7 & Caecum & 10 & Wound infection \\
\hline 4 & $\mathrm{~T}$ & DPL & AA & 5 & Small bowel & 4 & - \\
\hline 5 & $\mathrm{~T}$ & $\mathrm{DL}$ & $\mathrm{AA}$ & 3 & Small bowel & 6 & - \\
\hline 6 & $T$ & $\mathrm{DL}$ & $\mathrm{AA}+\mathrm{TA}$ & 3 & Stomach, diaphragm & 13 & Atelectesia \\
\hline 7 & $\mathrm{~T}$ & $\mathrm{DL}$ & $\mathrm{AA}+\mathrm{TA}$ & 4 & Small bowel & 4 & - \\
\hline 8 & $\mathrm{~T}$ & Endoscopy & $\mathrm{AA}$ & 4 & Stomach & 9 & - \\
\hline 9 & $\mathrm{~T}$ & $\begin{array}{l}\text { Sudden drop in } \\
\text { haematocrit value }\end{array}$ & AA & 2 & Abdominal aorta & 8 & - \\
\hline 10 & NT & Physical examination & AA & 1.5 & Liver & 8 & - \\
\hline 11 & $\mathrm{~T}$ & Physical examination & $A A$ & 6 & Right colon & 10 & Wound infection \\
\hline \multicolumn{8}{|c|}{ Delayed laparotomies } \\
\hline 1 & $\mathrm{~T}$ & DPL & AA & 14 & Small bowel & 4 & - \\
\hline 2 & $\mathrm{~T}$ & DPL & AA & 16 & Small bowel & 4 & - \\
\hline 3 & $\mathrm{~T}$ & DPL & $\mathrm{AA}$ & 9 & Small bowel, urinary bladder, ureter & 7 & Wound infection \\
\hline 4 & $\mathrm{~T}$ & DPL & $A A$ & 11 & Small bowel, omentum & 4 & - \\
\hline 5 & $\mathrm{~T}$ & DPL & $A A$ & 12 & Small bowel, transverse colon & 14 & Evisceration \\
\hline 6 & $\mathrm{~T}$ & DPL & $\mathrm{AA}$ & 8.5 & Small bowel & 4 & - \\
\hline 7 & $\mathrm{~T}$ & Physical examination & AA & 8.5 & Small bowel & 6 & - \\
\hline 8 & NT & Physical examination & $\mathrm{AA}$ & 15 & Transverse colon serosa & 5 & - \\
\hline 9 & $\mathrm{~T}$ & Physical examination & AA+Back & 13 & Left colon serosa, small bowel, & 9 & Wound infection \\
\hline 10 & NT & Physical examination & AA & 9.5 & Caecum serosa, mesocolon & 5 & - \\
\hline 11 & $\mathrm{~T}$ & $\mathrm{DL}$ & TA & 20 & Splenic flexura, diaphragm & 14 & Atelectesia \\
\hline 12 & $\mathrm{~T}$ & $\mathrm{DL}$ & AA & 9 & Right colon & 15 & - \\
\hline 13 & $T$ & $\mathrm{DL}$ & AA & 13 & Small bowel & 6 & - \\
\hline 14 & $\mathrm{~T}$ & $\mathrm{CT}$ & Back & 8.5 & Left colon & 10 & Wound infection \\
\hline
\end{tabular}


US showed no additional injury and thus the patient was discharged 72 hours later. Another patient with contrast extravasation from left colon on CT examination underwent surgery to repair a splenic flexure injury. This patient had initially refused colonoscopic examination on admission.

Echocardiography was performed for 11 patients who had left thoracoabdominal penetrating injuries. Pericardial effusion was detected only in one patient, who was managed with tube thoracostomy because of pneumothorax. DL showed no additional injury. Following his control echocardiography, the patient discharged 7 days after admission.

While colonoscopy was applied to 9 of the 17 patients with back region injuries, gastroscopy were applied to 8 of the 16 patients with epigastric injuries. The remaining patients did not give consent for this procedure. No injuries were found during colonoscopy, but gastroscopy revealed injury at the corpus of the stomach in one patient, which was repaired with gastroraphy as part of the early laparotomy group.

Although mean (SD) PATI scores were higher in the laparotomy group ( $12(2.1)$; range 4-33) than in the selective group (11 (2.4); range 4-28) there was no statistically significant difference in PATI between the operative and nonoperative groups $(\mathrm{p}>0.05)$. Mean $(\mathrm{SD})$ hospitalisation time was 120 (69) hours (median 96 hours) for patients who underwent laparatomy and 24.8 (12) hours (median 20 hours) for the patients who were managed non-operatively (Mann-Whitney U test, $\mathrm{p}<0.0001$ ). The mean duration between admission and laparotomy was 4.3 hours for the early group and 12 hours for the delayed group. There was no mortality because of delayed laparotomy, and morbidity, which was $32 \%$, was not due to delay of operations. Among all the patients, the most common complication was wound infection $(n=21,18 \%)$. The rates of morbidities of the early and delayed laparotomy groups were $27 \%$ and $36 \%$ respectively $(\mathrm{p}=0.1$, Fisher's exact test).

\section{DISCUSSION}

The stabbed patient with minimal or no symptoms after penetrating injury still poses a management challenge for trauma surgeons in the emergency department. There is no doubt that haemodynamic instability or any sign of peritoneal irritation warrants immediate laparotomy; however, the major challenge nowadays is to make the right decision about the diagnostic modalities available. Surgeons must decide which patients with penetrating abdominal stab wound requires laparotomy, when patients should be operated on, and which diagnostic procedure should be used. Unfortunately, a single evaluation test will never provide adequate diagnosis in all cases of penetrating abdominal trauma.

The goal of diagnosis and management should be to decrease the rate of unnecessary laparatomies, while identifying those in whom it is necessary. Mandatory laparotomy for truncal stab wounds leads to unnecessary surgery in 37$40 \%$ of patients. ${ }^{815}$ Complications of negative laparotomy may be severe; when performed for trauma, the mortality rate changes from $0 \%$ to $6 \%$, and negative laparotomy is associated with a 5-22\% complication rate. ${ }^{8-10} 15$ In a previous study, when exploration was a routine procedure, negative and nontherapeutic laparotomy rates were $12 \%$ and $23 \%$ respectively. ${ }^{13}$ In the latter period of the study, when selective management was the routine procedure, these rates decreased to $7 \%$ and $4 \% .{ }^{13}$ Non-therapeutic laparotomy rate in our study was $12 \%$, but with selective management combined with the diagnostic tests we were able to decrease the rate of negative laparotomy to $0 \%$.

The ongoing dilemma for haemodynamically stable patients almost came to a conclusion with the introduction of "selective conservatism" by Shaftan. ${ }^{1}$ One of his suggestions in his study in 1960 was that physical examination of the abdomen could reliably and safely predict the need for laparotomy for the patients with abdominal trauma. After performing standard resuscitative protocol as an initial approach for all the patients, our algorithm was based on serial physical examinations supported by white blood cell counts, axillary/rectal temperature measures, and other diagnostic techniques.

Authors from the Netherlands had guided their management for abdominal stab wounds by serial physical examinations. ${ }^{16}$ Even though they had used local wound exploration and DPL, these were abandoned in the latter period of their study. They decreased the rate of laparotomies for stab wounds from $55 \%$ to $30 \%$ and the rate of non-therapeutic laparotomies from $24 \%$ to $0 \%$, without increased mortality morbidity due to delayed laparotomy. In our study, two patients in the early laparotomy group and four in the delayed group underwent laparotomy after physical examination only. The rates of therapeutic and non-therapeutic laparotomies were equal in each group, and there was no negative laparotomy. However, we emphasise that physical examination should be combined with other diagnostic procedures.

The traditional recommendation was to operate on all patients with evisceration from the stab wound. ${ }^{517} 18$ Kimberly et al, in their prospective study, stated that laparatomy was necessary in $77 \%$ of patients with omental evisceration and $80 \%$ of patients with visceral evisceration. ${ }^{19}$ However, neither omental nor bowel evisceration was a mandatory indication for laparatomy in our study. All of the 16 omental and three small bowel eviscerations were replaced in the abdomen, and only one patient with eviscerated small bowel underwent laparatomy because of bile revealed by DL performed 16 hours after admission. Although there was a failure in non-operative treatment of cases with small bowel evisceration, we believe that this result is not significant because the number was very limited.

DPL has been the gold standard for the evaluation of blunt abdominal trauma over the past few decades, ${ }^{20}$ and in the 1980s, was adopted for the management of penetrating wounds. ${ }^{21} 22$ Thal published the first report on the selective management of abdominal stab wounds using peritoneal lavage. ${ }^{2}$ As the other diagnostic modalities developed, DPL was nearly abandoned as the initial approach. In a recent study, Gonzales et al concluded that DPL counts of $<1000$ red blood cells per $\mathrm{mm}^{3}$ identified patients who would have negative local wound exploration or insignificant abdominal stab wounds, so these patients could be safely sent home from the emergency department immediately. ${ }^{23}$

Although DPL was a routine procedure for all patients with peritoneal penetration in our institute, it was abandoned after 1998. ${ }^{13}$ During our study, DPL was applied to haemodynamically stable patients with uncertain peritonitis findings, unconsciousness due to associating head trauma, and/or progressive signs of peritonitis. The main reason we used DPL was to remove the blood seen during US from the peritoneal cavity by irrigating with isotonic saline via the inserted cannula, so patients could sustain serial physical examinations.

The concept of using DL in penetrating trauma is not new and was first reported by Carnevale et al in 1977. ${ }^{24}$ Another study showed that patients with multiple sites of abdominal penetration or isolated flank and thoracoabdominal injuries can benefit from an aggressive DL programme, and the authors of that study emphasised a decrease in the negative laparotomy rate. ${ }^{25}$ The role of DL in injuries of the anterior abdomen is limited; however, stab wounds of the thoracoabdominal region represent the most appropriate injuries for laparoscopic evaluation. ${ }^{26}{ }^{27}$ We recommend performing DL 
for injuries of the anterior abdomen when there is uncertainty regarding peritonitis without obvious indications for laparotomy, or in cases of omental or hollow viscus herniation for selected patients. It is also our policy to perform DL in all patients with left thoracoabdominal injuries so as to detect unsuspected diaphragmatic injuries. ${ }^{28}$ In our study, no negative or non-therapeutic laparotomies were performed following DL.

The use of US in blunt trauma has been routine since the 1980 s, ${ }^{29}$ and has a reported sensitivity of $81-88 \%$ and specificity of $97-100 \%{ }^{30-33}$ In some of the recent studies, the authors pointed out the benefits of FAST in penetrating abdominal trauma. ${ }^{34-36}$ In $50 \%$ of the FAST scans we performed, we were able to demonstrate some abnormalities, such as free fluid in abdominal cavity, spleen, liver, renal injuries and we could also sample the fluid for macroscopic and microscopic analysis under its guidance. However, FAST did not itself mandate laparotomy, but was rather used as an indicator of injuries that required surgery. Boulanger et al considered a positive sonogram to be a positive "noninvasive" DPL, ${ }^{30}$ while Udobi et al recommended the use of FAST early in the algorithm, but emphasised that it is not as reliable as in blunt trauma and has a $15 \%$ negative laparotomy rate. FAST should therefore be combined with other diagnostic modalities when selecting patients for laparotomy with penetrating abdominal trauma. ${ }^{34}$

Back injuries should not be evaluated by local wound exploration because of the thick paraspinal muscle in this region. Triple contrast CT and/or colonoscopy will give sufficient information about the damage. ${ }^{36-40}$ Even though it may be difficult to perform endoscopy in an unprepared colon, we at least perform sigmoidoscopy after rectal enema in cases with left sided injuries. In this study no injuries were detected by coloscopy, whereas gastroscopy revealed an injury in the gastric corpus that was repaired within 4 hours of admission. CT scan showed splenic flexura injury in one patient whose laparotomy was performed as part of the delayed group. Echocardiography revealed pericardial effusion but no cardiac injury in one patient in our study.

In conclusion, the use of physical examination alone and/ or together with different diagnostic methods allows reduction in nontherapeutic laparotomies and elimination of negative laparatomies. The surgeon has to make vital decisions for the appropriate choice among various diagnostic techniques for non-operative management of penetrating abdominal stab wound.

\section{Authors' affiliations}

C Ertekin, H Yanar, K Taviloglu, R Güloglu, Department of General Surgery, Istanbul Faculty of Medicine, Istanbul University, Istanbul, Turkey

O Alimoglu, Department of General Surgery, Vakif Gureba Training Hospital, Istanbul, Turkey

Competing interests: none declared

\section{REFERENCES}

1 Shaftan GW. Indications for operation in abdominal trauma. Am J Surg 1960;99:657-64.

2 Thal ER. Evaluation of peritoneal lavage and local exploration in lower chest and abdominal stab wounds. J Trauma 1977;17:642-8.

3 McAlvananah MJ, Shaftan GW. Selective concervatism in penetrating abdominal stab wounds: a continuing reappraisal. J Trauma 1978;18:206-12.

4 Demetriades D, Rabinowitz B. Indications for operation in abdominal stab wounds. A prospective study of 651 patients. Ann Surg 1987;205:129-32.

5 Leppaniemi AK, Haapiainen RK. Selective nonoperative management of abdominal stab wounds: prospective randomized study. World I Surg 1996;20:1101-6.
6 Bostrom L, Heinius G, Nilsson B. Trends in the incidence and severity of stab wounds in Sweden 1987-1994. Eur J Surg 2000;166:765-70.

7 Forde KA, Ganepola GAP. Is mandatory exploration for penetrating abdominal trauma extinct? The morbidity and mortality of negative exploration in a large municipal hospital. J Trauma 1974; 14:764-6.

8 Sirinek KR, Page CP, Root HD, et al. Is exploratory celiotomy necessary for all patients with truncal stab wounds? Arch Surg 1990;125:844-8.

9 Lowe RJ, Boyd DR, Folk FA, et al. The negative laparotomy for abdominal trauma. J Trauma 1972;12:853-61.

10 Thompson JS, Moore EE, van Duzer-Moore S, et al. The evolution of abdominal stab wound management. J Trauma 1980;20:478-84.

11 Demetriades D, Vandenbossche P, Ritz M, et al. Nontherapeutic operations for penetrating trauma: early morbidity and mortality. Br J Surg 1993;80:860-1.

12 American College of Surgeons. Advanced trauma life suppor ${ }^{\circledR}$, Chicago, 1997.

13 Taviloglu K, Gunay K, Ertekin C, et al. Abdominal stab wounds: the role of selective management. Eur J Surgery 1998;164:17-21.

14 Moore EE, Dunn EL, Moore JB, et al. Penetrating abdominal trauma index. $J$ Trauma 1981;21:439-45.

15 Leppaniemi A, Salo J, Haapiainen R. Complications of negative laparotomy for truncal stab wounds. J Trauma 1995;38:54-8.

16 van Haarst EP, van Bezooijen BP, Coene PP, et al. The efficacy of serial physical examination in penetrating abdominal trauma. Injury 1999;30:599-604.

17 Oreskovich MR, Carrico CJ. Stab wounds of the anterior abdomen: analysis of a management plan using local wound exploration and quantitative peritoneal lavage. Ann Surg 1983;193:411-19.

18 Thanvendran A, Vijayaragavan A, Rasaretnam R. Selective surgery for abdominal stab wounds. Br J Surg 1975;62:750-2.

19 Kimbery N, Roxanne R, Kimberly J, et al. Evisceration after abdominal stab wound: Is laparotomy required? J Trauma 1999;47:622-7.

20 Root HD, Keizer PJ, Perry JF Jr. Peritoneal trauma, experimental and clinical studies. Surgery 1967;62:679-86.

21 Thompson JS, Moore EE. Perioneal lavage in the evaluation of penetrating abdominal trauma. Surg Gynecol Obstet 1981;153:861-3.

22 Goldenberg JH, Bernstein DM, Rodman GH, et al. Selection of patients with abdominal stab wounds for laparotomy. J Trauma 1982;2:476-80.

23 Gonzalez RP, Turk B, Falimirski ME, et al. Abdominal stab wounds: diagnostic peritoneal lavage criteria for emergency room discharge. J Trauma 2001;51:939-43

24 Carnevale N, Baron N, Delany HM. Peritoneoscopy as an aid in the diagnosis of abdominal trauma: a preliminary report. J Trauma 1977;17:634-41.

25 Simon RJ, Rabin J, Kuhls D. Impact of increased use of laparoscopy on negative laparotomy rates after penetrating trauma. J Trauma 2002; 53:297-302.

26 Ivatury RR, Simon RJ, Weksler B, et al. Laparoscopy in the evaluation of the intrathoracic abdomen after penetrating injury. J Trauma 1992;33:101-9.

27 DeMaria EJ, Dalton JM, Gore DC, et al. Complementary roles of laparoscopic abdominal exploration and diagnostic peritoneal lavage for evaluating abdominal stab wounds: a prospective study. J Laparoendosc Adv Surg Tech 2000;10:131-6.

28 Ertekin C, Guloglu R, Onaran Y, et al. The use of laparoscopy as a primary diagnostic and therapeutic method in penetrating wounds of lower thoracal region. Surgical Laparosc Endosc 1998;8:26-9.

29 Gruessner R, Mentges B, Duber C, et al. Sonography versus peritoneal lavage in blunt abdominal trauma. J Trauma 1989;29:242-4.

30 Boulanger BR, Brenneman FD, Kirkpatrick AW, et al. The indeterminate abdominal sonogram in multisystem blunt abdominal trauma. J Trauma 1998:45:52-6.

31 Bode PJ, Edwards MJR, Kruit MC, et al. Sonography in a clinical algorithm for early evaluation of 1671 patients with blunt abdominal. AJR Am J Roentgenol 1999;172:905-11.

32 Rozycki GS, Ochsner MG, Schmidt JA, et al. A prospective study of surgeonperformed ultrasound as the primary adjuvant modality for injured patient assessment. J Trauma 1995;39:492-500.

33 McKenney MG, Martin L, Lentz, et al. 1,000 consecutive ultrasounds for blunt abdominal trauma. J Trauma 1996;40:607-12.

34 Udobi KF, Rodriguez A, Chiu WC, et al. The role of ultrasonography (FAST) in penetrating abdominal trauma. J Trauma 2001;50:475-9.

35 Bernard RB, Kearney PA, Tsuei B, et al. The routine use of sonography in penetrating torso injury is beneficial. J Trauma 2001;51:320-5.

36 Soto JA, Morales C, Munera F, et al. Penetrating stab wounds to abdomen: use of serial US and contrast-enhanced CT in stable patients. Radiology $2001 ; 220: 365-71$.

37 Albrecht RM, Vigil A, Schermer CR, et al. Stab wounds to the back/flank in hemodynamically stable patients: evaluation using triple-contrast computed tomography. Am J Surg 1999;65:683-7.

38 Ivatury RR, Ochsner MG, Simon R, et al. Cavitary endoscopy. In: Ivatury RR, Cayten CG, eds. The textbook of penetrating trauma. Baltimore: Williams \& Wilkins, 1996:281-99.

39 Simon RJ, lvatury RR. Current concepts in the use of cavitary endoscopy in the evaluation and treatment of blunt and penetrating truncal injuries. Surg Clin North Am 1995;75:157-74.

40 Taviloglu K. When to operate on abdominal stab wounds. Scand J Surg 2002;91:58-61. 